\title{
Liaison stable entre un canal potassique et le récepteur des sulfonylurées
}

De nombreux tissus et, plus particulièrement, les muscles lisses et squelettiques et la cellule $\beta$ pancréatique sont le siège de courants potassiques sensibles à l'ATP. Dans la cellule $\beta$ pancréatique, les canaux $\mathrm{K}^{+}$sensibles à l'ATP $\left(\mathrm{K}_{\mathrm{ATP}}\right)$, inhibés par l'ATP, sont impliqués dans la régulation de la sécrétion d'insuline par le glucose et sont la cible des sulfonylurées, molécules hypoglycémiantes utilisées dans le traitement de certains diabètes non insulinodépendants [1]. Le clonage récent du gène codant pour le récepteur des sulfonylurées de rat et de hamster (désigné SUR) montre que cette protéine de 1 582 acides aminés est constituée de multiples domaines transmembranaires et de deux régions liant potentiellement les nucléotides [1]. L'importance de ces sites est évidente, la délétion du second site étant responsable d'une maladie familiale rare appelée PHHI (persistent hyperinsulinemic hypoglycemia of infancy), caractérisée par une altération de l'homéostasie du glucose due à l'absence de régulation de la sécrétion d'insuline. De par sa structure et sa relation probable avec un canal ionique spécifique, SUR représente un nouveau membre de la famille des protéines ABC (ATP binding cassette), famille dont font partie les produits des gènes CFTR (cystic fibrosis transmembrane conductance regulator) et MDR (multidrug resistance) [2]. La fonction régulatrice du transport de chlorure et de sodium transépithélial par la protéine CFTR est aujourd'hui très partiellement élucidée. Ainsi, il est admis que la protéine CFTR sert de transporteur pour l'ATP cellulaire qui, sécrété dans le milieu extracellulaire, interagit avec le récepteur puri- nergique $\mathrm{P}_{2 \mathrm{U}}$ dont l'activation induit l'ouverture d'un canal chlorure spécifique ou ORCC (outward-rectifying Cl-channel) $\left(\mathrm{m} / \mathrm{s} n^{\circ} 11\right.$, vol. 11, p. 1612). En outre, la protéine CFTR semble aussi impliquée dans la régulation de l'activité de canaux sodiques épithéliaux. Dans le cas de MDR, sa capacité de transporter l'ATP et de contrôler un autre canal chlorure (volume-activated Cl-channel) font de cette protéine un autre exemple de mode de régulation complexe à laquelle participent un canal ionique, une protéine membranaire transportant l'ATP et probablement un accepteur d'ATP. La sensibilité aux sulfonylurées des $\mathrm{K}_{\mathrm{ATP}}$ de la cellule $\beta$ pancréatique et la grande homologie de SUR avec les membres de la famille des protéines $A B C$ suggèrent que le récepteur SUR et le canal $\mathrm{K}_{\mathrm{ATP}}$ de la cellule $\beta$ pancréatique sont fonctionnellement associés. C'est ce qui vient d'être démontré par une équipe japonaise qui rapporte le clonage du gène codant pour une protéine désignée BIR ( $\beta$ cell inward rectifier) qui, lorsqu'elle est cotransfectée avec SUR dans les cellules COS, engendre une activité de courant potassique sensible à l'ATP [3]. C'est chez l'homme que le gène codant pour la protéine BIR (hBIR) a été initialement cloné; ce gène a ensuite servi à cloner celui de la souris à partir d'une lignée de cellules $\beta$ pancréatiques $(\mathrm{m} B I R)$. La protéine, de 390 acides aminés, est très proche de la protéine $\mathrm{uK}_{\mathrm{ATP}}-1$ de rat, membre de la famille des small inward rectifiers, rectificateurs par courants entrants de faible amplitude. La présence de motifs d'acides aminés conservés dans tous les canaux potassiques rectificateurs par courants entrants et, en particulier, la localisation spécifique d'une asparagine dans la séquence protéique de BIR suggèrent fortement que BIR est un faible rectificateur. L'ARN messager de BIR a pu être détecté dans tous les tissus qui expriment des courants potassiques sensibles à l'ATP, en quantité importante dans les îlots pancréatiques et dans plusieurs lignées de cellules $\beta$ pancréatiques et en quantité moindre dans le cœur, le muscle squelettique et le cerveau. De façon surprenante, la distribution de l'ARN messager de la protéine SUR coïncide tout à fait avec celle de BIR, et cela dans les mêmes proportions. Plus surprenant encore, le gène codant pour la protéine BIR se trouve localisé sur le bras court du chromosome 11, immédiatement en aval du gène codant pour SUR. Alors que dans des cellules COS transfectées avec mBIR ou avec haSUR (SUR de hamster), seuls des courants potassiques insensibles à l'ATP ont pu être détectés, la cotransfection des deux gènes reconstitue une conductance $\mathrm{K}^{+}$sensible à l'ATP dont les caractéristiques sont tout à fait représentatives d'un courant $\mathrm{K}^{+}$rectificateur de faible amplitude. Le glibenclamide, bloqueur de canaux potassiques, ferme le canal reconstitué alors que le diazoxide, qui ouvre ce canal dans la cellule $\beta$ pancréatique, stimule l'activité du canal $\mathrm{K}^{+}$dans les cellules COS recombinantes. L'efficacité de la reconstitution du canal $\mathrm{K}^{+}$sensible à l'ATP est confirmée par sa capacité de transport ionique. En effet, le flux sortant basal de rubidium, qui reflète l'activité d'un canal $\mathrm{K}^{+}$, est inhibé par le glibenclamide et activé par le diazoxide. Enfin, un troisième modèle utilisant des ovocytes de Xenopus lae- 
vis dans lesquels $\mathrm{mBIR}$ et haSUR ont été injectés confirme la reconstitution fonctionnelle d'un courant $\mathrm{K}^{+}$ sensible au glibenclamide et au diazoxide dans la membrane ovocytaire. Ces résultats démontrent pour la première fois que les protéines BIR et SUR reconstituent les principales caractéristiques du courant $\mathrm{K}^{+}$sensible à l'ATP décrit dans la cellule $\beta$ pancréatique. Ce canal est donc constitué de deux sous-unités, BIR ou $\mathrm{K}_{\text {ATP }}-\alpha$ et SUR ou $\mathrm{K}_{\mathrm{ATP}}-\beta$. Il est suggéré que la sous-unité $K_{\text {AтP }}-\beta$ active la sous-unité $\mathrm{K}_{\mathrm{ATP}}-\alpha$, c'est-à-dire le canal $\mathrm{K}^{+}$rectificateur interne silencieux, et lui confère la sensibilité à l'ATP et aux sulfonylurées. La stœchiométrie de ces sous-unités, le devenir de l'ATP liant SUR, le site de liaison pour le diazoxide sont quelques-unes des grandes inconnues de ce modèle. Cette reconstitution apporte des bases moléculaires solides et déterminantes pour la compréhension du fonctionnement des courants ioniques impliqués dans le désordre de l'homéostasie du glucose.

B.A.

1. Ladzunski M. Les canaux potassiques sensibles à l'ATP ou les suites imprévues de l'étude des sulfamides hypoglycémiants. médecine/sciences 1990; $6: 279-85$.

2. Aguilar-Bryan L, Nichols CG, Wechsler SW, Clement JP, Boyd AE, Gonzales G, Herrera-Sosa H, Nguy K, Bryan J, Nelson DA. Cloning of the beta cell high affinity sulfonylurea receptor: a regulator of insulin secretion. Science 1995; 268: 423-6. 3. Lepage P, Gros P. La glycoprotéine P: de la résistance croisée aux médicaments au transport des lipides biliaires. médecine/sciences 1995; 11 : 357-66.

4. Inagaki N, Gonoi T, Clement IV JP, Namba N, Inazawa J, Gonzales G, Aguilar-Bryan L, Seino S, Bryan J. Reconstitution of $\mathrm{IK}_{\mathrm{ATP}}$ : an inward rectifier subunit plus the sulfonylurea receptor. Science 1995; 270 : 1166-9. 